\title{
A Comparative Study Between Dexmedetomidine As An Adjunct To Bupivacine In Comparison To Bupivacine Alone In Haemodynamic Stability
}

\author{
Prof. Dr Subrata Nag, Dr. Ritesh Pandey
}

\begin{abstract}
Spinal anaesthesia is one of the most common procedure used in clinical anaesthesia practice. It has the advantage that profound nerve block can be produced in a large part of the body by the relatively simple injection of a small amount of local anaesthetic. Hypotension is one of the most common event encountered with the procedure Hypotension and bradycardia during spinal anesthesia are common and may relate to severe adverse events such as cardiac arrest or death. Preventive measures include fluid preload, lateral tilt, and use of vasopressors. Search is still on for the pharmacological agents that can provide hemodynamic stability with neuraxial blockade.
\end{abstract}

\section{Objective}

The prolongation of spinal anesthesia by using opioids and $\alpha 2$ agonist like Clonidine through the oral,intravenous and spinal route has been known. The new $\alpha 2$ agonist, dexmedetomidine has also been proved to prolong the spinal anesthesia through the intrathecal route.The aim of the study is to compare dexmedetomidine as an adjunct to bupivacaine in comparison to bupivacine alone \& its effect on various haemodynamic factors.

Methods

Fourty six patients of ASA grade I and II between 18-58yrs of age of either sex, admitted in RIMS,RANCHI (Jharkhand), scheduled for elective lower abdominal \& lower limb surgical procedure were included The study protocol was approved by Institutional Ethical Committee and written informed consent was obtained from all patients.

The pre-anaesthetic check-up included a detailed medical and surgical history, and any previous anaesthetic exposure with its outcome. General examination includes general condition, built, weight, pulse rate, blood pressure, respiratory rate, and presence of cyanosis, anaemia, clubbing, jaundice or edema. A careful thorough systemic examination was done to rule out any cardiovascular, respiratory, gastrointestinal and neurological or any other systemic illness.Routine biochemistry investigation included haemoglobin, total leucocyte count, differential leucocyte count, blood sugar, blood urea, and serum creatinine, were done in all patients. ECG and $\mathrm{X}$-Ray Chest were done in patients where indicated and in those over 40 years of age along with other relevant investigation.

After taking detailed history and thorough clinical examination, the patients were excluded from the study on the basis of below mentioned criteria:

Patients with systemic hypertension, hepatic dysfunction, renal dysfunction, endocrine dysfunction, cardiac dysfunction, morbid obesity (body weight more than $20 \%$ of the ideal body weight), Other exclusion criteria were patients with known drug hypersensitivity, those on antihypertensive medication or antidepressant drugs and those who refused to give consent.

- Inclusions

- ASA I \& II

- 18-58 yrs of age of either sex

- $\quad$ Scheduled for elective lower abdominal \& lower limb surgical procedure

- Exclusions

- Contraindications to spinal anesthesia

- Allergy to local anesthetics

- Systemic hypertension

- Hepatic, renal, endocrine, cardiac dysfunction

- Morbid obesity (body weight more than $20 \%$ of the ideal body weight

- Patients on antihypertensive medication or antidepressant drugs

- Patients who refused to give consent. 
Patients using 2-adrenergic receptors antagonists, calcium channel blockers, angiotensin converting enzyme inhibitors, or noted to have dysrhythmias on the electrocardiogram(ECG), a body weight of more than $120 \mathrm{~kg}$, or height less than $150 \mathrm{~cm}$ were excluded from the study. Standard monitoring was used, including noninvasive arterial blood pressure, ECG, heart rate (HR) and pulseoximetry (Spo2).

The total 46 patients were randomly divided into two groups of 23 patients each according to a computer generated random table. Group B $(\mathrm{n}=80)$ patients received $15 \mathrm{mg}$ of bupivacaine \& $1 \mathrm{ml}$ of NS and Group D $(\mathrm{n}=80)$ patients received bupivacane $15 \mathrm{mg} \& 10 \mu \mathrm{g}$. Group allocation was done by an assistant who was unaware of the study protocol and was not involved in the study.

- The total 46 patients were randomly divided into two groups of 23 patients each.

\section{$15 \mathrm{mg}+1 \mathrm{ml}$ of NS}

- Group B - inj $0.5 \%$ hyperbaric bupivacaine

- Group D - inj $0.5 \%$ hyperbaric bupivacaine

\section{$15 \mathrm{mg}+1 \mathrm{ml}$ of Dexmedetomidine}

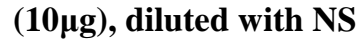

Total volume of Drug was $4 \mathrm{ml}$ in both the groups.

\section{Anaesthetic technique}

Patients were premedicated with tab. alprazolam $0.25 \mathrm{mg}$ and tab. Ranitidine $150 \mathrm{mg}$ the night before the surgery. All patients were kept fasting for 8 hours prior to surgery.

On arrival to operation theatre routine monitoring was started and base line vital parameters of heart rate, systemic arterial pressure including systolic, diastolic and mean arterial pressure, arterial oxygen saturation (SpO2), and ECG were recorded. An intravenous line was secured and Ringer lactate was given at rate of 6-8 $\mathrm{ml} / \mathrm{kg}$. All patients received premedication of intravenous Inj. Midazolam $(0.02 \mathrm{mg} / \mathrm{kg})$ and inj. glycopyrrolate $(0.01 \mathrm{mg} / \mathrm{kg})$..

After preparation with the patient in sitting position spinal anaesthesia was performed at L3-L4 level through a midline approach with quincke needle. Study group B patients were given bupivacaine $15 \mathrm{mg} \& 1 \mathrm{ml}$ of NS (total volume $4 \mathrm{ml}$ ) and group D bupivacaine $15 \mathrm{mg} \& 10 \mu \mathrm{g}$ dexmedetomidine(total volume $4 \mathrm{ml}$ ). Study medication was prepared by an anaesthesiologist who was blinded to the randomization schedule.the anaesthesiologist performing block recorded baseline vitals preoperatively,every 3 min for first 15 min,then every 5 min until patient discharged from PACU.sensory dermatome was assessed by pinprick \& motor by modified bromage score

\section{Bromage Scale For Motor Blockade}

\begin{tabular}{|ll|}
\hline I & Free movement of hips, legs and feet \\
\hline II & Just able to flex knees with free movement of feet \\
\hline III & Unable to flex knees, but with free movement of feet \\
\hline IV & Unable to move hips or legs or feet \\
\hline
\end{tabular}

The Hemodynamic changes observed as abnormal findings during the study were defined as follows :

- Hypotension was defined as SBP $<20 \%$ of baseline value or $<90 \mathrm{~mm} \mathrm{Hg}$, whichever was lower.

- Hypertension was defined as SBP > 20\% of baseline value or $>140 \mathrm{~mm} \mathrm{Hg}$ whichever was higher.

- Tachycardia was defined as heart rate $>100 /$ minute.

- Bradycardia was defined as heart rate $<50 /$ minute

After completion of surgery patients transferred to post anaesthesia care unit and monitored for any hemodynamic changes or any other adverse effects.

\section{Results}

- The data recorded in tabulated manner and was analyzed using Microsoft Excel and SPSS software version 16.0 for windows.

- Statistical analysis done using independent student ' $t$ ' test for parametric data and Chi square test for nonparametric variables.

- A 'p' value of less than 0.05 was considered statistically significant.

Out of 46 patients one patient from group B \& two from group D were considered as failure.43 patients completed study protocol and included in data.the onset of block \& duration is given in Table 1.The various vitals monitoring before during and after procedure are arranged in table 2 . 
Table 1.

Gender

Group $b(n=21)$

Group D(n=22)

Male

12

14

Female

9

Surgery

Abdominal \& vaginal

hystrectomy 8

Appendicitis 5

Hernia 7

Turp

Skin grafting

8
7
1

6

8

2

0

4

2

Demographic parameters

\begin{tabular}{|l|l|l|l|}
\hline & Group D $(\mathbf{n = 2 3})$ & Group B $(\mathbf{n = 2 3})$ & p value \\
\hline $\begin{array}{l}\text { Age }(\mathrm{yrs}) \\
\mathrm{Sex}(\mathrm{M} / \mathrm{F})\end{array}$ & $32.54 \pm 10.73$ & $34.07 \pm 12.03$ & 0.39 \\
\hline $\mathrm{Ht}(\mathrm{cm})$ & $149.73 \pm 4.76$ & $11 / 12$ & 0.273 \\
\hline $\mathrm{Wt}(\mathrm{Kg})$ & $55.8 \pm 8.41$ & $149.03 \pm 5.77$ & 0.61 \\
\hline $\begin{array}{l}\text { ASA grade } \\
\text { I/II }\end{array}$ & $19 / 4$ & $53.6 \pm 9.44$ & 0.28 \\
\hline
\end{tabular}

Various surgical interventions done in patients in both groups

\begin{tabular}{|l|l|l|}
\hline \multicolumn{1}{|c|}{ Surgery } & $\begin{array}{l}\text { Group } \\
\text { D }\end{array}$ & Group B \\
\hline Appendectomy & 4 & 2 \\
\hline Hernia & 8 & 9 \\
\hline Hysterectomy & 7 & 7 \\
\hline DHS fixation & 2 & 3 \\
\hline Skin grafting & 0 & 1 \\
\hline Turp & 2 & 1 \\
\hline
\end{tabular}

\section{Comparative evaluation of Mean Systolic Blood Pressure (mm Hg)}

\begin{tabular}{|r|l|l|l|}
\hline $\begin{array}{c}\text { Time } \\
\text { interval in min }\end{array}$ & Group D & Group B & Pvalue \\
\hline Base & $137.19 \pm 13.18$ & $134.79 \pm 14.62$ & 0.14 \\
\hline $\begin{array}{l}3 \text { min after spinal } \\
\text { anesthesia }\end{array}$ & $131.06 \pm 13.09$ & $130.56 \pm 13.07$ & 0.40 \\
\hline 6 min & $127.80 \pm 13.74$ & $126.34 \pm 12.25$ & 0.24 \\
\hline $9 \min$ & $125.98 \pm 15.79$ & $124.66 \pm 19.44$ & 0.33 \\
\hline $12 \min$ & $123.22 \pm 14.78$ & $121.52 \pm 13.49$ & 0.22 \\
\hline $15 \min$ & $119.32 \pm 15.72$ & $118.92 \pm 12.60$ & 0.09 \\
\hline $30 \mathrm{~min}$ & $116.21 \pm 12.40$ & $116.43 \pm 12.20$ & 0.40 \\
\hline $45 \mathrm{~min}$ & $115.68 \pm 10.94$ & $116.10 \pm 10.45$ & 0.08 \\
\hline $60 \mathrm{~min}$ & $112.80 \pm 10.40$ & $115.16 \pm 10.70$ & 0.16 \\
\hline $75 \mathrm{~min}$ & $112.78 \pm 10.01$ & $114.39 \pm 10.35$ & 0.40 \\
\hline $90 \mathrm{~min}$ & $113.65 \pm 7.71$ & $113.34 \pm 8.14$ & 0.30 \\
\hline Postop & $115 \pm 7.64$ & $114.39 \pm 7.34$ & 0.45 \\
\hline erative & & & \\
\hline
\end{tabular}


Comparative evaluation of Mean Systolic Blood Pressure ( $\mathrm{mm} \mathrm{Hg}$ )

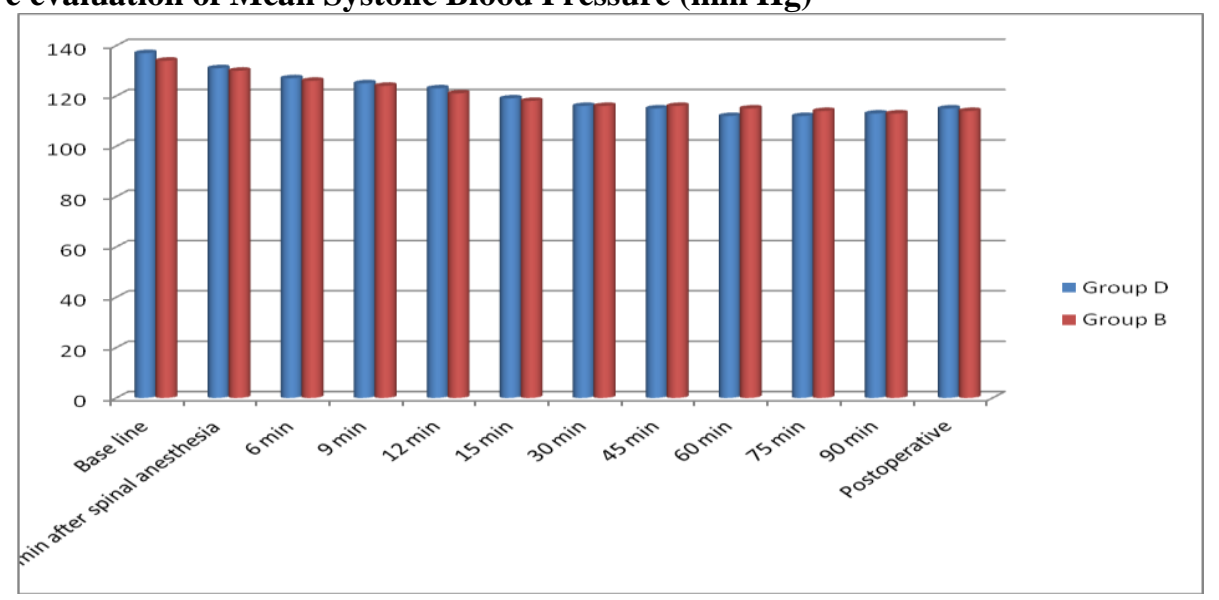

Comparative evaluation of Mean Diastolic Blood Pressure ( $\mathrm{mm} \mathrm{Hg}$ )

\begin{tabular}{|c|c|c|c|}
\hline Time interval in min & Group D & Group B & P-value \\
\hline Base line & $85.85 \pm 9.07$ & $86.08 \pm 9.99$ & 0.43 \\
\hline $\begin{array}{l}3 \text { min after spinal } \\
\text { anesthesia }\end{array}$ & $82.59 \pm 7.86$ & $79.61 \pm 9.40$ & $0.01^{*}$ \\
\hline 6 min & $80.93 \pm 7.78$ & $77.26 \pm 10.33$ & $0.006^{* *}$ \\
\hline 9 min & $80.21 \pm 8.18$ & $75.38 \pm 9.68$ & $0.0005^{* *}$ \\
\hline 12 min & $78.52 \pm 8.21$ & $73.61 \pm 9.15$ & $0.0003^{* *}$ \\
\hline 15 min & $75.69 \pm 10.89$ & $73.28 \pm 8.38$ & $0.04 *$ \\
\hline 30 min & $74.57 \pm 8.29$ & $72.43 \pm 8.47$ & $0.05^{*}$ \\
\hline 45 min & $74.52 \pm 8.59$ & $71.33 \pm 8.32$ & $0.01^{*}$ \\
\hline 60 min & $73.44 \pm 7.59$ & $70.53 \pm 6.98$ & $0.007^{* *}$ \\
\hline 75 min & $72.88 \pm 6.95$ & $72.67 \pm 6.96$ & 0.42 \\
\hline 90 min & $71.86 \pm 6.77$ & $73.05 \pm 6.37$ & 0.13 \\
\hline Postoperative & $72.48 \pm 6.52$ & $73.64 \pm 6.47$ & 0.13 \\
\hline
\end{tabular}

Comparative evaluation of Mean Diastolic Blood Pressure (mm Hg)

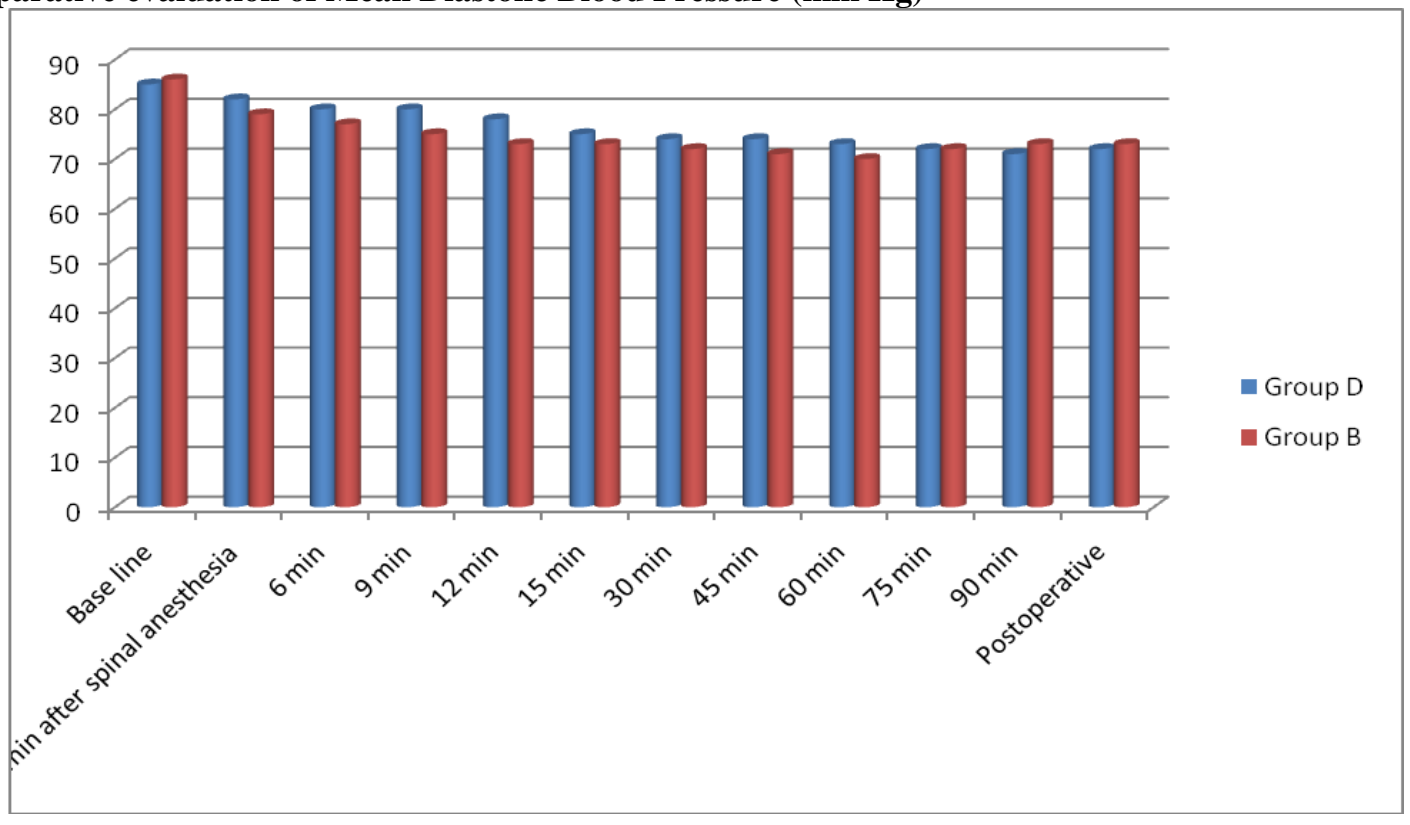




\section{Comparative evaluation of Mean Arterial blood pressure (mm Hg)}

\begin{tabular}{|l|l|l|l|}
\hline $\begin{array}{l}\text { Time interval in } \\
\text { min }\end{array}$ & Group D & Group B & P -value \\
\hline Base line & $103.02 \pm 9.49$ & $102.11 \pm 10.55$ & 0.28 \\
\hline $\begin{array}{l}3 \text { min after spinal } \\
\text { anesthesia }\end{array}$ & $98.86 \pm 8.88$ & $95.82 \pm 9.59$ & $0.024 *$ \\
\hline 6 min & $96.56 \pm 9.25$ & $93.23 \pm 9.63$ & $0.01 *$ \\
\hline 9 min & $95.47 \pm 9.86$ & $91.30 \pm 9.93$ & 0.07 \\
\hline 12 min & $93.40 \pm 9.72$ & $88.58 \pm 9.52$ & $0.001 * *$ \\
\hline 15 min & $90.26 \pm 12.00$ & $88.14 \pm 8.75$ & 0.10 \\
\hline 30 min & $88.51 \pm 9.05$ & $87.62 \pm 9.10$ & 0.27 \\
\hline 45 min & $88.30 \pm 8.74$ & $86.93 \pm 8.68$ & 0.16 \\
\hline 60 min & $86.42 \pm 7.89$ & $85.25 \pm 7.18$ & 0.47 \\
\hline 75 min & $85.81 \pm 5.97$ & $86.33 \pm 7.49$ & \\
\hline Postoperative & $86.5 \pm 5.61$ & $87.17 \pm 5.55$ & \\
\hline
\end{tabular}

Comparative evaluation of Mean Arterial blood pressure (mm Hg)

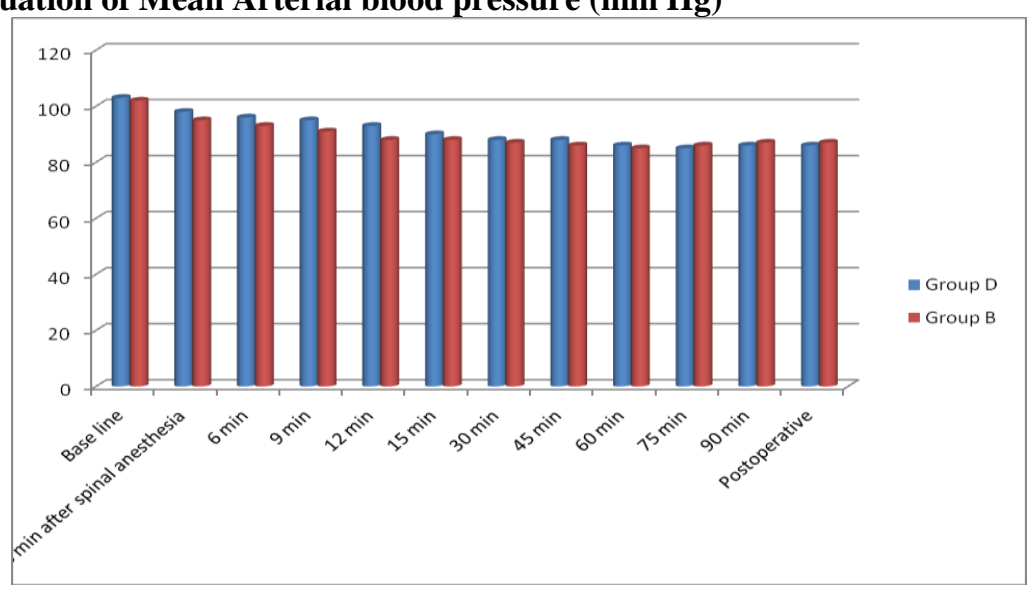

\section{Comparative evaluation of Mean Heart rate (beats/min)}

\begin{tabular}{|l|l|l|l|}
\hline $\begin{array}{l}\text { Time interval in } \\
\text { min }\end{array}$ & Group D & Group B & P -value \\
\hline Base line & $100.55 \pm 11.47$ & $88.03 \pm 11.57$ & 1.0 \\
\hline $\begin{array}{l}1 \text { min after spinal } \\
\text { anesthesia }\end{array}$ & $97.98 \pm 10.05$ & $88.08 \pm 11.06$ & 1.0 \\
\hline 3 min & $96.60 \pm 9.17$ & $82.87 \pm 11.74$ & 0.17 \\
\hline 5 min & $95.06 \pm 10.02$ & $74.67 \pm 10.51$ & $0.0^{* *}$ \\
\hline $10 \mathrm{~min}$ & $92.68 \pm 9.53$ & $72.29 \pm 10.69$ & $0.0^{* *}$ \\
\hline $15 \mathrm{~min}$ & $90.23 \pm 12.08$ & $69.21 \pm 9.41$ & $0.0^{* *}$ \\
\hline $30 \mathrm{~min}$ & $87.68 \pm 8.91$ & $68.61 \pm 10.02$ & $0.0^{* *}$ \\
\hline $45 \mathrm{~min}$ & $86.98 \pm 8.79$ & $66.70 \pm 9.09$ & $0.0^{* *}$ \\
\hline $60 \mathrm{~min}$ & $85.48 \pm 8.30$ & $65.92 \pm 8.06$ & $0.0^{* *}$ \\
\hline $75 \mathrm{~min}$ & $86 \pm 8.59$ & $64.91 \pm 7.41$ & $0.0^{* *}$ \\
\hline $90 \mathrm{~min}$ & $84.05 \pm 6.07$ & $65.34 \pm 7.06$ & $0.0^{* *}$ \\
\hline Postoperative & $83.67 \pm 5.44$ & $66.38 \pm 5.94$ & $0.0^{* *}$ \\
\hline
\end{tabular}




\section{Comparative evaluation of Mean Heart rate (beats/min)}

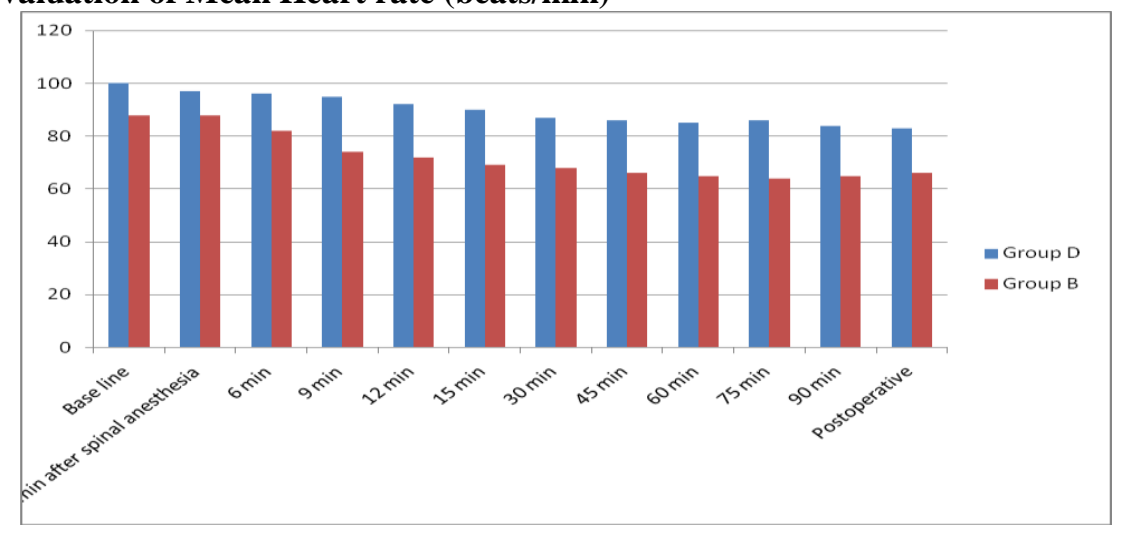

\section{Intra-operative and postoperative adverse events}

\begin{tabular}{|l|l|l|}
\hline & Group D & Group B \\
\hline Adverse Events & No. of patient & No. of patient \\
\hline Hypotension & 2 & 8 \\
\hline Bradycardia & 1 & 3 \\
\hline Shivering & 1 & 5 \\
\hline Headache & 0 & 0 \\
\hline Nausea & 0 & 2 \\
\hline
\end{tabular}

\section{Conclusion}

- Dexmedetomidine when used as an adjunct to bupivacaine in spinal anaesthesia helped in keeping the patient hemodynamically stable throughout the surgery.

- Intrathecal dexmedetomidine did not potentiate the effect of bupivacaine on blood pressure. This may be explained by the mechanism local anaesthetics affect blood pressure. Local anaesthetics reduce blood pressure by decreasing sympathetic outflow. Sympathetic blockade produced by intrathecal dexmedetomidine does not decrease blood pressure further presumably because the blockade produced by bupivacaine is nearly maximum.

- The intrathecal use of dexmedetomidine is off label. Highest dose of intrathecal dexmedetomidine used in animal studies was $100 \mu \mathrm{g}$.Konakci and colleagues reported white matter injury in rats when high dose epidural dexmedetomidine $(6 \mu \mathrm{g} / \mathrm{kg})$ was used alone; however,

- subsequently Brummett and co-workers demonstrated no injury and a protective effect when doses of 26$40 \mu \mathrm{g} / \mathrm{kg}$ were used perineurally.

- In humans the largest epidural dose used was $2 \mu \mathrm{g} / \mathrm{kg}$ (and the largest intrathecal dose used was $10 \mu \mathrm{g}$.

- Although no major neurological complications have been reported so far, larger studies are required to rule out any short term or long term adverse effects.

\section{References}

[1]. Hala E A Eid MD, Mohamed A Shafie MD, Hend Youssef MD. Dose-Related Prolongation of Hyperbaric Bupivacaine Spinal Anesthesia by Dexmedetomidine Ain Shams Journal of Anaesthesiology, Cairo, Egypt

[2]. Liu S, McDonald S. Current issues in spinal anesthesia. Anesthesiology 2001; 94: 888-906.

[3]. Al- Ghanem S M., Massad IM., AlMustafa M M. , Al- Zaben K R., Qudaisat I Y, Qatawneh A M, AbuAli H M. Effect of Adding Dexmedetomidine versus Fentanyl to Intrathecal Bupivacaine on Spinal Block Characteristics in Gynecological Procedures: A Double Blind Controlled Study American Journal of Applied Sciences, 20096 (5): 882-887 